\title{
Host antitumor immune response activated by Dual POLA1-HDAC11 inhibitors endowed with a large spectrum of antitumor activity
}

Claudio Pisano, Lucio Merlini, Sergio Penco, Raffaella Cincinelli, Nadine Darwiche, Mario B. Guglielmi, Ilaria La Porta, Maddalena Pizzulo, Federica Prosperi, Giacomo Signorino, Fabiana Colelli, Francesco Cardile, Laura Focareta, Alessandra Fucci, Egildo L. D'Andrea, Assunta Riccio and Sabrina Dallavalle

\section{Abstract}

Small molecule modulators of chromatin modifying enzymes became the focus of drug discovery efforts, because of their direct antitumor effects, such as apoptosis and cell-cycle arrest, in addition to their indirect immunomodulation properties (Peedicayil, 2012; West and Johnstone, 2014; Falkenberg and Johnstone, 2014). Recent studies have shown that inhibition of HDACs or POLA1 activity exerts anti-proliferative effects and also potentiates the immune response by activating type I Interferon (Medon et al., 2017; Starokadomskyy et al, 2016). To exploit a potential synergy, hybrid molecules targeting simultaneously POLA1 and HDACs, were designed. Here, we present in vitro and in vivo data concerning the antitumor activity of new dual POLA1-HDAC11 inhibitors. Among a library of screened molecules, MIR002 and MIR144 showed an antiproliferative activity at nanomolar concentrations on a panel of human tumor cell lines. In vitro functional assays revealed that these molecules potently inhibit POLA1 and specifically act on HDAC11 that recently emerged as regulator of several immune cells differentiation, pointing out its immunomodulatory role. Mechanistically, MIR002 and MIR144 induce acetylation of Histone $\mathrm{H} 4$ and alphaTubulin, canonical targets of HDACs, and p53-K382, as well. Activation of p53 leads to p21 increase and cell cycle arrest in the G1/S boundary. These compounds could be even more effective in treating human cancers when they are appropriately combined with other chemotherapeutics, such as Cisplatin. Indeed, the combination of MIR002 or MIR144 with Cisplatin, revealed a synergistic interaction (Combination Index <1) on NCI-H460 and H460-R9A (ST1926-resistant sub-line) cell growth. The in vivo oral administration of MIR002 or MIR144 showed a potent antitumor activity, with a Tumor Growth Inhibition comprised between 60 and $100 \%$ in a series of human and murine solid and hematological tumors (human MM473 and MM432 Pleural Mesothelioma, H460 and H460R9A NSCLC, A2780-Dx and A2780-DDP ovarian carcinomas, NB-4 Acute Promyelocitic leukemia and Murine EL-4 Lymphoma) xenografted in nude and immunocompetent mice. The strongest antitumor effect of MIR144 was observed in immunocompetent mice rather than in nude mice suggesting that an immunomodulatory effect, along with the direct antiproliferative activity, contributes to tumor inhibition. Further analysis confirmed that MIR144 antitumor activity involves the induction/activation of several components of the host immune system, both at humoral and cellular levels. The large spectrum of antitumor activity, together with the high tolerability observed, open the possibility for their clinical investigation in different population of cancer patients. 